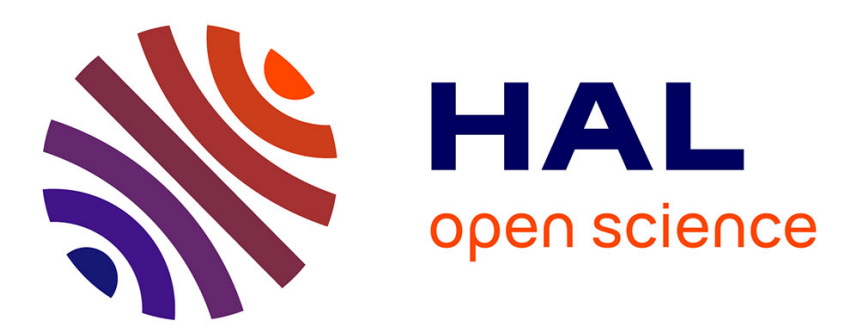

\title{
Uniform Global Position Feedback Tracking Control of Mechanical Systems Without Friction
}

Antonio Loria

\section{To cite this version:}

Antonio Loria. Uniform Global Position Feedback Tracking Control of Mechanical Systems Without Friction. 2013 IEEE American Control Conference ACC, Jun 2013, Washington, DC, United States. pp.5722-5727, 10.1109/ACC.2013.6580734 . hal-00831449

\section{HAL Id: hal-00831449 \\ https://hal.science/hal-00831449}

Submitted on 7 Jun 2013

HAL is a multi-disciplinary open access archive for the deposit and dissemination of scientific research documents, whether they are published or not. The documents may come from teaching and research institutions in France or abroad, or from public or private research centers.
L'archive ouverte pluridisciplinaire HAL, est destinée au dépôt et à la diffusion de documents scientifiques de niveau recherche, publiés ou non, émanant des établissements d'enseignement et de recherche français ou étrangers, des laboratoires publics ou privés. 


\title{
Uniform Global Position Feedback Tracking Control of Mechanical Systems Without Friction
}

\author{
Antonio Loría
}

\begin{abstract}
We establish, as far as we know, the first proof of uniform global asymptotic stability for a mechanical system (Euler-Lagrange) in closed loop with a dynamic controller which makes use only of position measurements. The controller is fairly simple, it is reminiscent of the so-called PadenPanja controller [20] where unavailable generalized velocities are replaced by approximate differentiation (dirty derivatives). The controller has been reported previously however, only semiglobal ${ }^{1}$ asymptotic stability has been established so far. The novelty of this paper relies in establishing a global property as well as in the method of proof, which does not follow Lyapunov's. However, the problem of finding a strict control Lyapunov function remains open.
\end{abstract}

\section{INTRODUCTION}

We study Euler-Lagrange systems, given by the equation

$$
D(q) \ddot{q}+C(q, \dot{q}) \dot{q}+g(q)=u
$$

where $q \in \mathbb{R}^{n}$ denotes the generalized positions, $\dot{q}$ denotes the generalized velocities, $D: \mathbb{R}^{n} \rightarrow \mathbb{R}^{n \times n}$ corresponds to the inertia matrix function, $C: \mathbb{R}^{n} \times \mathbb{R}^{n} \rightarrow \mathbb{R}^{n \times n}$ corresponds to the Coriolis and centrifugal forces matrix, $g: \mathbb{R}^{n} \rightarrow \mathbb{R}^{n}$ represents the vector of forces which are derived from the potential energy function $U: \mathbb{R} \rightarrow \mathbb{R}$ i.e., $g(q):=\frac{\partial U}{\partial q}(q)$ and $u \in \mathbb{R}^{n}$ is the vector of control inputs. All functions are smooth in their arguments.

We revisit the problem of output-feedback tracking control, which consists in designing a dynamic controller with output $u$ that makes use of $q$ as the only plant measurement and ensures that given a smooth bounded trajectory $t \mapsto q_{d}$ the generalized coordinates satisfy

$$
\lim _{t \rightarrow \infty} q(t) \rightarrow q_{d}(t), \quad \lim _{t \rightarrow \infty} \dot{q}(t) \rightarrow \dot{q}_{d}(t) .
$$

More precisely (and of much higher difficulty) the problem consists in establishing uniform global asymptotic stability of the origin of the closed-loop system. We put special emphasis on the qualifier global which implies that the property must hold for all initial states of the closed-loop system, including the tracking errors in $\mathbb{R}^{2 n}$ as well as the controller's states. Not to be confused with terminologies such as "global on the set $\mathcal{X} \subset \mathbb{R}^{n}$ " or the weaker property "global in the plant's variables and semi-global in the controller's".

In the last 30 years or so there have been numerous attempts to solve the problem mentioned above, as a particular paradigm of dynamic output feedback control of

The author is with CNRS. Address: LSS-SUPELEC, 91192 Gif-surYvette, France. E-mail: antonio.loriaelss.supelec.fr.

${ }^{1}$ That is, the domain of attraction may be arbitrarily enlarged by enlarging the control gains. nonlinear systems. See for instance [16] and other works by the same authors on output feedback linearization that are collected in [15]. In a similar train of thought we find methods that rely on the ability to perform a coordinate transformation of system (1) into models that are linear in the unmeasured velocities. See for instance the work of G. Besançon -[1], [2] and subsequent references, including [13]. However, it has been long recognized that such transformations are inapplicable to many physical systems; even to simple two-degrees-of-freedom planar robots with revolute joints - see [25]. In the article [11] we intend to circumvent the difficulties encountered in preceding literature by exploring a change of coordinates which yields a nonminimal realization. However, the resulting controller (for the system of augmented dimension) is not implementable via output-feedback since it must satisfy a mechanical constraint whose verification requires the knowledge of the unmeasured velocities. During the preparation of this final manuscript we became aware of [23] where the author presents a global result for Hamiltonian systems which relies on a clever but intricate observer-design and a change of coordinates that involves the computation of the square root of $D(q)^{-1}$.

Other works focus on robot tracking control. For instance, the classic paper [4] presented a proof of uniform asymptotic stability using Kelly's controller [8] originally proposed for set-point control. The author in [4] invokes Tychonov's theorem on singularly perturbed systems to show uniform global asymptotic stability provided that the unique pole of the dirty-derivatives filter used in [8] is placed at $-\infty$ that is, the result actually establishes semi-global asymptotic stability. The same property is achieved via Lyapunov's direct method in [12]. Relying on the practically reasonable but theoretically restrictive assumption that the system possesses (natural) viscous friction which induces damping, the authors in [18] established global asymptotic stability. That is, the model considered is

$$
D(q) \ddot{q}+C(q, \dot{q}) \dot{q}+F \dot{q}+g(q)=u
$$

where $F$ is symmetric positive definite. However, under these conditions, it is direct to extend the stability property from semi-global to global, for a number of results in the literature.

To the best of the author's knowledge it has not been established either, that uniform for system (1) global asymptotic stability via output feedback is out of reach. What is more, it rather seems that the absence of proof or disproof has simply eluded the efforts of many researchers throughout 
the years and is not due to a structural impediment. This is investigated in the seminal article [17] where the concept of unboundedness observability is introduced. Roughly, from the main results in [17] it may be concluded that the system

$$
d \ddot{q}+c \dot{q}^{2}=u, \quad q, u \in \mathbb{R}
$$

cannot be stabilized globally by dynamic output feedback with output $q$. The obstacle is that the system does not possess the unboundedness observability property that is, the solution $[\dot{q}(t), q(t)]$ may escape to infinity even for bounded values of $q(t)$. Notice that this is not the case of Lagrangian systems which possess the structural property of skew-symmetry of the matrix $\overparen{D(q)}-2 C(q, \dot{q})$. Indeed, uniform global asymptotic stability of systems

$$
d(q) \ddot{q}+c(q) \dot{q}^{2}+g(q)=u
$$

is established in [10] provided that $\overparen{d(q)}=2 c(q) \dot{q}^{2}$. As a matter of fact this is probably the only article that presents a dynamic output-feedback controller for Euler-Lagrange systems together with a strict Lyapunov function albeit for one-degree-of-freedom systems. The extension to the case of $n$-degree-of-freedom systems has not been obtained yet: attempts include [27], [3] however, the controller from [27] is guaranteed (in the non-adaptive case, only) to achieve uniform asymptotic stability for any system's initial conditions provided that the controller's trajectories lay in a forwardinvariant set. Yet, the result in [27] relies on the restrictive assumption that the model includes viscous friction (of known magnitude in the non-adaptive case) i.e., as in (2) and that the forces derived from potential energy are bounded. The controller of [3] is not implementable without velocity measurements.

In summary, to the best of the author's knowledge establishing uniform global asymptotic stability remains open; roughly speaking, there are two types of results addressing this problem. Those based on Lyapunov's direct method and those which intended to exploit structural properties. In the first case, stability is global only with respect to part of the states -as in [27] or is semiglobal -as in [4], [12] etc. In the second case, the structural assumptions needed to perform convenient changes of coordinates do not hold for EL systems $-c f$. [25].

The rest of the paper is organized as follows. For the sake of clarity we recall basic stability definitions in Section II. In Section III we present our main result and in Section IV.

\section{PRELIMINARIES}

To remove all possible ambiguity we start by recalling a few definitions of stability from [6] and some statements which are either known or are re-stated in an original manner, for the purposes of this article. Consider the dynamic system

$$
\dot{x}=f(t, x), \quad x \in \mathbb{R}^{n}, t \in \mathbb{R}_{\geq 0} .
$$

We denote by $x\left(t, t_{\circ}, x_{\circ}\right)$ or when the context is clear by $x(t)$, the solutions of (3) with initial times $t_{\circ} \in \mathbb{R}_{\geq 0}$ and initial states $x_{\circ} \in \mathbb{R}$ that is, we have $x\left(t_{\circ}, t_{\circ}, x_{\circ}\right)=x_{\circ}$. Recall that a continuous function $\alpha: \mathbb{R}_{\geq 0} \rightarrow \mathbb{R}_{\geq 0}$ is of class $\mathcal{K}$ if it is strictly increasing and $\alpha(0)=0$, a continuous function $\sigma: \mathbb{R}_{\geq 0} \rightarrow \mathbb{R}_{\geq 0}$ is of class $\mathcal{L}$ if it is strictly decreasing and $\sigma(s) \rightarrow 0$ as $s \rightarrow \infty$; a continuous function $\beta: \mathbb{R}_{\geq 0} \times \mathbb{R}_{\geq 0} \rightarrow \mathbb{R}_{\geq 0}$ is of class $\mathcal{K} \mathcal{L}$ if $\beta(r, \cdot) \in \mathcal{L}$ and $\beta(\cdot, s) \in \mathcal{K}$; a continuous function $\alpha: \mathbb{R}_{\geq 0} \rightarrow \mathbb{R}_{\geq 0}$ is of class $\mathcal{K}_{\infty}$ if $\alpha \in \mathcal{K}$ and $\alpha(s) \rightarrow \infty$ as $s \rightarrow \infty$. We denote by $|\cdot|$, the Euclidean norm of vectors (or any other compatible norm) and the induced norm of matrices.

Definition 1 (Uniform global boundedness) The solutions of (3) are said to be uniformly globally bounded if there exist $\gamma \in \mathcal{K}_{\infty}$ and $c>0$ such that, for all $\left(t_{\circ}, x_{\circ}\right) \in \mathbb{R}_{\geq 0} \times \mathbb{R}^{n}$ each solution $x\left(\cdot, t_{\circ}, x_{\circ}\right)$ satisfies

$$
\left|x\left(t, t_{\circ}, x_{\circ}\right)\right| \leq \gamma\left(\left|x_{\circ}\right|\right)+c \quad \forall t \geq t_{\circ} .
$$

Note that for any $r$ there exists $R$ independent of $t_{\circ}$ such that $\left|x_{\circ}\right| \leq r$ implies that $\left|x\left(t, t_{\circ}, x_{\circ}\right)\right| \leq R$. This property is commonly established via auxiliary functions.

Theorem 1 Let $V: \mathbb{R}_{\geq 0} \times \mathbb{R}^{n} \rightarrow \mathbb{R}_{\geq 0}$ be continuously differentiable; $\alpha_{1}, \alpha_{2}$ be functions of class $\mathcal{K}_{\infty}$ and let $a \in \mathbb{R}$ and $c>0$ be such that

$$
\begin{array}{cl}
\alpha_{1}(|x|) \geq V(t, x) \geq \alpha_{2}(|x|)+a & \forall(t, x) \in \mathbb{R}_{\geq 0} \times \mathbb{R}^{n} \\
\dot{V}(t, x):=\frac{\partial V}{\partial t}+\frac{\partial V}{\partial x} f(t, x) \leq 0 & \forall t \in \mathbb{R}_{\geq 0}, x:|x| \geq c
\end{array}
$$

Then, the solutions of (3) are uniformly globally bounded.

The following definition may be found in [6].

Definition 2 (Uniform global stability) The origin of system (3) is said to be uniformly globally stable if there exists $\gamma \in \mathcal{K}_{\infty}$ such that for each $\left(t_{\circ}, x_{\circ}\right) \in \mathbb{R}_{\geq 0} \times \mathbb{R}^{n}$, each solution $x\left(\cdot, t_{\circ}, x_{\circ}\right)$ satisfies

$$
\left|x\left(t, t_{\circ}, x_{\circ}\right)\right| \leq \gamma\left(\left|x_{\circ}\right|\right) \quad \forall t \geq t_{\circ} .
$$

Note that uniform global stability tantamounts to uniform stability plus uniform global boundedness.

Theorem 2 Let the conditions of Theorem 1 hold for $a=$ $c=0$. Then, the origin of (3) is uniformly globally stable. If the conditions hold only in an open neighborhood of the origin with $\alpha_{1}, \alpha_{2} \in \mathcal{K}$, the latter is uniformly stable.

Proof. The global statement: following the proof of Theorem 1 we have $|x(t)| \leq \alpha_{2}^{-1} \circ\left(\alpha_{1}\left(\left|x_{\circ}\right|\right)\right)$. The local statement is due to Persidskii -[22] and appears in numerous textbooks.

Definition 3 (Uniform global attractivity) The origin of system (3) is said to be uniformly globally attractive if for each $r, \sigma>0$ there exists $T>0$ such that

$$
\left|x_{\circ}\right| \leq r \Longrightarrow\left|x\left(t, t_{\circ}, x_{\circ}\right)\right| \leq \sigma \quad \forall t \geq t_{\circ}+T .
$$

Proof. The proof follows along similar lines as [26, Theorem 25, p. 165].

\section{Definition 4 (Uniform Global Asymptotic Stability)}

The origin of system (3) is said to be uniformly globally asymptotically stable if it is 
- uniformly stable;

- the solutions are uniformly globally bounded;

- the origin is uniformly globally attractive.

It is important to emphasize that only all three conditions in Definition 4 together, imply the existence of a class $\mathcal{K} \mathcal{L}$ function $\beta$ such that the solutions of (3) satisfy

$$
|x(t)| \leq \beta\left(\left|x_{\circ}\right|, t-t_{\circ}\right) \quad \forall t \geq t_{\circ} \geq 0
$$

which leads to the construction of converse Lyapunov functions and in turn, implies robustness with respect to external perturbations but the latter cannot be concluded from uniform stability plus uniform global attractivity alone. Whence the importance of uniform global boundedness in nonlinear timevarying systems.

The following statement establishes uniform global asymptotic stability using integral conditions, in the spirit of Barbălat's lemma however, we recall that the latter may not be used to establish uniform convergence.

Lemma 1 [21] Let $F: \mathbb{R}_{\geq 0} \times \mathbb{R}^{n} \rightarrow \mathbb{R}^{n}$ be continuous. If the origin of the system $\dot{x}=F(t, x)$ is uniformly globally stable and there exists a continuous positive definite function $\gamma: \mathbb{R}^{n} \rightarrow \mathbb{R}_{\geq 0}$ and for each $r, \nu>0$ there exists $\beta_{r \nu}>0$, such that for all $\left(t_{\circ}, x_{\circ}\right) \in \mathbb{R}_{\geq 0} \times B_{r}$, all solutions $x\left(\cdot, t_{\circ}, x_{\circ}\right)$ and all $t \geq t_{\circ}$,

$$
\int_{t_{\circ}}^{t}\left[\gamma\left(x\left(\tau, t_{\circ}, x_{\circ}\right)\right)-\nu\right] d \tau \leq \beta_{r \nu}
$$

then the origin of the system $\dot{x}=F(t, x)$ is uniformly globally asymptotically stable.

\section{MAIN RESULT}

The following assumptions are fairly standard in the literature of robot control but are also satisfied by a number of Euler-Lagrange systems such as electrical and electromechanical -see [19], as well as some marine systems -see [5]. In particular, these hypotheses hold for robot manipulators composed of revolute joints only or prismatic joints only.

\section{Assumption 1}

1) There exist positive real numbers $d_{m}$ and $d_{M}$ such that

$$
d_{m} \leq|D(q)| \leq d_{M}, \quad \forall q \in \mathbb{R}^{n} ;
$$

2) there exists $k_{c}>0$ such that

$$
\begin{aligned}
& |C(x, y)| \leq k_{c}|y| \\
& C(x, y) z=C(x, z) y \\
& \forall x, y \in \mathbb{R}^{n} \\
& \forall x, y, z \in \mathbb{R}^{n} ;
\end{aligned}
$$

3) the matrix $\overparen{D(q)}-2 C(q, \dot{q})$ is skew symmetric.

Definition 5 (global output-feedback tracking control) Consider the EL system (1). Suppose that only position measurements are available and that the properties enumerated in Assumption 1 hold. Furthermore, assume that the reference trajectory $t \mapsto q_{d}$ is of class $\mathcal{C}^{2}$ and that there exists $k_{\delta}>0$ such that

$$
\max \left\{\sup _{t \geq 0}\left|q_{d}(t)\right|, \sup _{t \geq 0}\left|\dot{q}_{d}(t)\right|, \sup _{t \geq 0}\left|\ddot{q}_{d}(t)\right|\right\} \leq k_{\delta} .
$$

Under these conditions, find a dynamic output-feedback controller

$$
\begin{aligned}
\dot{q}_{c} & =f\left(t, q_{c}, q\right) \\
u & =u\left(t, q_{c}, q\right)
\end{aligned}
$$

such that the closed-loop system

$$
\begin{gathered}
D(q) \ddot{q}+C(q, \dot{q}) \dot{q}+g(q)=u\left(t, q_{c}, q\right) \\
\dot{q}_{c}=f\left(t, q_{c}, q\right)
\end{gathered}
$$

has a unique equilibrium at

$$
\begin{gathered}
\left(q_{c}-q_{c}^{*}, \tilde{q}, \dot{\tilde{q}}\right)=(0,0,0), \\
\tilde{q}:=q-q_{d}(t), \quad \dot{\tilde{q}}:=\dot{q}-\dot{q}_{d}(t)
\end{gathered}
$$

where $q_{c}^{*}$ is a solution to (10) with $q \equiv q_{d}$, which is uniformly globally asymptotically stable.

Theorem 3 Consider the system (1) under Assumption 1. Let $a, b, k_{p}$ and $k_{d}$ be positive constants satisfying

$$
\begin{aligned}
& \min \left\{\frac{k_{p}}{4 d_{M}}, \frac{k_{d}}{4 b d_{M}}\right\}>1 \\
& k_{b}\left(k_{d}-k_{c} k_{b} k_{\delta}\right)>k_{c} k_{\delta} .
\end{aligned}
$$

where $k_{b}:=a / b$ and consider the dynamic position-feedback controller

$$
\begin{aligned}
\dot{q}_{c} & =-a\left(q_{c}+b \tilde{q}\right) \\
\vartheta & =q_{c}+b \tilde{q} \\
u & =-k_{p} \tilde{q}-k_{d} \vartheta+D(q) \ddot{q}_{d}+C\left(q, \dot{q}_{d}\right) \dot{q}_{d}+g(q)
\end{aligned}
$$

Then, there exist $a^{*}$ and $b^{*}$ independent of the initial conditions such that if $a \geq a^{*}, b \geq b^{*}$ the origin $\{z=0\}$ with $z:=\left[\tilde{q}^{\top}, \dot{\tilde{q}}^{\top}, q_{c}^{\top}\right]^{\top}$ is uniformly globally asymptotically stable.

We show that there exist minimal values of the filter parameters $a$ and $b$ such that, provided that the controller gains satisfy (12), the origin is uniformly asymptotically stable for any initial conditions in $t_{\circ},\left[\tilde{q}\left(t_{\circ}\right), \dot{\tilde{q}}\left(t_{\circ}\right), q_{c}\left(t_{\circ}\right)\right] \in \mathbb{R}_{\geq 0} \times$ $\mathbb{R}^{3 n}$. However, the proof is (unfortunately) not constructive in the sense that we do not use Lyapunov's direct method.

Remark 1 We stress that the controller (13) corresponds to that from [12] where semiglobal asymptotic stability is established. The controller is also reminiscent of that published in [4] whose author relies on singular perturbation theory to establish that the domain of attraction may be extended to $\mathbb{R}^{3 n}$ provided that $a \rightarrow \infty$.

\section{A. Proof of Theorem 3}

The closed-loop equations are obtained by replacing $u$ from (13c) in (1) and subtracting $C\left(q, \dot{q}_{d}\right) \dot{q}$ to both sides of $(13 c)$ hence,

$$
D(q) \ddot{\tilde{q}}+\left[C(q, \dot{q})+C\left(q, \dot{q}_{d}\right)\right] \dot{\tilde{q}}+k_{p} \tilde{q}+k_{d} \vartheta=0 .
$$

Now, for the purpose of analysis we differentiate (13b) and use (13a) to obtain

$$
\dot{\vartheta}=-a \vartheta+b \dot{\tilde{q}}
$$


Note that the point $[\tilde{q}, \dot{\tilde{q}}, \vartheta]=[0,0,0]$ is an equilibrium of (14), (15) and is unique. Then, a direct computation shows that $\left[\tilde{q}, \dot{\tilde{q}}, q_{c}\right]=[0,0,0]$ is a unique equilibrium of the closedloop equations (13a), (14).

The stability proof is divided in three main steps which establish the three properties listed in Definition 4. Uniform stability trivially follows using Lyapunov's first method; it is also implicitly contained in the proof of the main result in [12] as well as in the proof of Proposition 2, below. Proposition 1 below, establishes uniform global boundedness and Proposition 2 establishes uniform global attractivity.

Proposition 1 The closed-loop trajectories of the system (1), (13) under the conditions of Theorem 3 are uniformly globally bounded.

Proof. We analyze the solutions to (14), (15) with initial conditions $t_{\circ} \geq 0$ and $x\left(t_{\circ}\right)=x_{\circ} \in B_{r}$ where $r>0$ is arbitrarily fixed.

First, we consider the Lyapunov function candidate

$$
V_{1}(t, \tilde{q}, \dot{\tilde{q}}, \vartheta)=\frac{1}{2}\left(\dot{\tilde{q}}^{\top} D\left(\tilde{q}+q_{d}(t)\right) \dot{\tilde{q}}+k_{p}|\tilde{q}|^{2}+\frac{k_{d}}{b}|\vartheta|^{2}\right)
$$

which, under Assumption 1 and in view of (14), (15), satisfies

$$
\begin{aligned}
\dot{V}_{1} & =-\frac{k_{d} a}{b}|\vartheta|^{2}+\dot{\tilde{q}}^{\top} C\left(q, \dot{q}_{d}\right) \dot{\tilde{q}} \\
& \leq-\frac{k_{d} a}{b}|\vartheta|^{2}+k_{c} k_{\delta}|\dot{\tilde{q}}|^{2} .
\end{aligned}
$$

Next, we introduce the following statement which follows as a corollary of [7, Theorem 2].

Lemma 2 Consider the differential equation

$$
\dot{\vartheta}(t)=-a \vartheta(t)+\nu(t), \quad \nu: \mathbb{R}_{\geq 0} \rightarrow \mathbb{R}^{n}, t \geq 0
$$

where $\nu(t)$ is uniformly continuous and bounded. Let $\tau>$ $t_{\circ}>0$ and $\epsilon:=1 / a$; if $\nu$ is uniformly continuous then

$$
\lim _{\epsilon \rightarrow 0} \dot{\vartheta}(t)=0
$$

uniformly on $[\tau, \infty)$.

We apply Lemma 2 to the equation (15) with $\nu(t)=b \dot{\tilde{q}}(t)$. To that end, we observe that (17) implies, by Assumption 1.1), that there exists $c>0$ such that $\dot{V}_{1} \leq c V_{1}$. Integrating the latter we obtain that the trajectories are defined on $\left[t_{\circ}, \infty\right)$ for any $t_{\circ} \geq 0$ therefore, for any $t^{\max }>t_{\circ}$ and $r$ there exists $M$ such that $\max \{|x(t)|,|\dot{x}(t)|\} \leq M\left(t^{\max }, r\right)$ for all ${ }^{2}$ $t \in\left[t_{\circ}, t^{\max }\right)$ and all $x_{\circ} \in B_{r}$. Therefore, $\nu(t)$ is bounded and uniformly continuous on $\left[t_{\circ}, t^{\mathrm{max}}\right)$. Now, the expression (19) implies that for any $\Delta>0$ there exists $a^{*}$ such that

$$
a \geq a^{*}(\Delta) \Rightarrow|\dot{\vartheta}(t)| \leq \Delta, \quad \forall t \in\left[\tau, t^{\max }\right)
$$

We emphasize that $a^{*}$ depends on $\Delta$ only and the latter may be chosen independent of $M$ (hence, independent of $r$ ) since the rate of convergence of $\dot{\vartheta}$ in (19) is independent of the bound on $\nu(t)$ and is uniform in $t$-see [7]. Let $\Delta=\Delta^{*}$

\footnotetext{
${ }^{2}$ The inequality for $|\dot{x}(t)|$ follows under Assumption 1
}

generate $a^{*}$ via (20). From (15) we see that for any $t \in$ $\left[\tau, t^{\max }\right)$ and $a \geq a^{*}$,

$$
b|\dot{\tilde{q}}(t)| \leq \Delta^{*}+a|\vartheta(t)| .
$$

Hence, defining $v_{1}(t):=V_{1}(t, \tilde{q}(t), \dot{\tilde{q}}(t), \vartheta(t))$ we obtain, from (17),

$$
\dot{v}_{1}(t) \leq-\frac{a}{b}\left(k_{d}-\frac{a k_{c} k_{\delta}}{b}\right)|\vartheta(t)|^{2}+k_{c} k_{\delta} \frac{\Delta^{* 2}}{b^{2}}
$$

for all $t \in\left[\tau, t^{\max }\right)$ and $a \geq a^{*}$. Let $b^{*} \geq \Delta^{*}$ then, for any $b \geq b^{*}$

$$
\dot{v}_{1}(t) \leq-k_{b}\left(k_{d}-k_{c} k_{\delta} k_{b}\right)|\vartheta(t)|^{2}+k_{c} k_{\delta} .
$$

Now, assume that $|x(t)| \rightarrow \infty$ as $t \rightarrow \infty$ then, either $|\vartheta(t)|$ grows unboundedly as $t \rightarrow \infty$ or it remains bounded. In the first case, since (21) holds for any $t^{\max }$ by continuity of solutions and since $\Delta$ is independent of $t^{\max }$ we can (if necessary) extend the interval so that for sufficiently large $t \in\left[\tau, t^{\max }\right)$ we have $|\vartheta(t)| \geq 1$ so in view of $(12 \mathrm{~b})$, $\dot{v}_{1}(t) \leq 0$ which implies that $v_{1}(t)$ is bounded. Since $V_{1}$ is radially unbounded we also obtain that $|x(t)|$ is uniformly bounded. Next, assume that $|\vartheta(t)|$ is uniformly bounded for any $t$ then, and either $|\dot{\tilde{q}}(t)|$ or $|\tilde{q}(t)|$ (or both) grow unboundedly. If $|\dot{\tilde{q}}(t)|$ grows unboundedly it follows, in view of (15), that $|\vartheta(t)| \rightarrow \infty$ and the previous reasoning applies again. Finally, consider the case that $|x(t)| \rightarrow \infty$ due to the unbounded growth of $|\tilde{q}(t)|$ and consider the function $V_{2}: \mathbb{R}_{\geq 0} \times \mathbb{R}^{3 n} \rightarrow \mathbb{R}_{\geq 0}$,

$$
V_{2}(t, \tilde{q}, \dot{\tilde{q}}, \vartheta)=\left(\varepsilon_{1} \tilde{q}-\varepsilon_{2} \vartheta\right)^{\top} D\left(\tilde{q}+q_{d}(t)\right) \dot{\tilde{q}}, \quad \varepsilon_{1}, \quad \varepsilon_{2}<1
$$

which in view of (14) and (15), satisfies

$$
\begin{aligned}
\dot{V}_{2}= & \left(\varepsilon_{1} \tilde{q}-\varepsilon_{2} \vartheta\right)^{\top}\left(-k_{d} \vartheta-k_{p} \tilde{q}-\left[C(q, \dot{q})+C\left(q, \dot{q}_{d}\right)\right] \dot{\tilde{q}}\right) \\
& +\varepsilon_{1} \dot{\tilde{q}}^{\top} D(q) \dot{\tilde{q}}-\varepsilon_{2}(-a \vartheta+b \dot{\tilde{q}})^{\top} D(q) \dot{\tilde{q}} \\
& +\left(\varepsilon_{1} \tilde{q}-\varepsilon_{2} \vartheta\right)^{\top} \overparen{D(q)} \dot{\tilde{q}} .
\end{aligned}
$$

Let $R$ be an arbitrary positive number and define

$$
\Omega:=\left\{x \in \mathbb{R}^{3 n}: \tilde{q} \in \mathbb{R}^{n}, \max \{|\dot{\tilde{q}}|,|\vartheta|\} \leq R\right\} .
$$

Then,

$$
\begin{aligned}
& \left|\varepsilon_{1} \tilde{q}^{\top} C(q, \dot{q})^{\top} \dot{\tilde{q}}\right| \leq \varepsilon_{1}|\tilde{q}||\dot{\tilde{q}}| k_{c}\left(R+k_{\delta}\right) \\
& \left|\varepsilon_{2} \vartheta^{\top} C(q, \dot{q})^{\top} \dot{\tilde{q}}\right| \leq \varepsilon_{2}|\vartheta||\dot{\tilde{q}}| k_{c}\left(R+k_{\delta}\right)
\end{aligned}
$$

-see (9). Under these conditions, note that the right-hand side of (24) may be upper bounded by a first-order polynomial of $|\tilde{q}|$ with coefficients which depend on $|\dot{\tilde{q}}|$ and $|\vartheta|$ which are bounded for all $x \in \Omega$. Therefore, using Assumption 1 and (25) we see that there exist positive numbers $c_{1}, c_{2}$ such that, defining $v_{2}(t):=V_{2}(t, \tilde{q}(t), \dot{\tilde{q}}(t), \vartheta(t))$,

$$
\dot{v}_{2}(t) \leq-\varepsilon_{1} k_{p}|\tilde{q}(t)|^{2}+c_{1}|\tilde{q}(t)|+c_{2}
$$

for all $t \geq t_{\circ}$ and $x(t) \in \Omega$ that is, $\dot{v}_{2}(t)$ becomes negative as $|\tilde{q}(t)| \rightarrow \infty$.

Next, define $V: \mathbb{R}_{\geq 0} \times \mathbb{R}^{3 n} \rightarrow \mathbb{R}$,

$$
V(t, x):=V_{1}(t, \tilde{q}, \dot{\tilde{q}}, \vartheta)+V_{2}(t, \tilde{q}, \dot{\tilde{q}}, \vartheta)
$$


which is positive definite for sufficiently large control gains, independently of the initial conditions. To see this, note that

$$
\begin{aligned}
V(t, x)=\frac{1}{2}\left(\begin{array}{c}
\tilde{q} \\
\dot{\tilde{q}}
\end{array}\right)^{\top}\left(\begin{array}{cc}
k_{p} I & \varepsilon_{1} D \\
\varepsilon_{1} D^{\top} & \frac{1}{2} D
\end{array}\right)\left(\begin{array}{c}
\tilde{q} \\
\dot{\tilde{q}}
\end{array}\right) \\
+\frac{1}{2}\left(\begin{array}{c}
\vartheta \\
\dot{\tilde{q}}
\end{array}\right)^{\top}\left(\begin{array}{cc}
\frac{k_{d}}{b} I & -\varepsilon_{2} D \\
-\varepsilon_{2} D^{\top} & \frac{1}{2} D
\end{array}\right)\left(\begin{array}{c}
\vartheta \\
\dot{\tilde{q}}
\end{array}\right)
\end{aligned}
$$

where both matrices are positive definite respectively if

$$
\frac{k_{p}}{4 d_{M}}>\varepsilon_{1}^{2}, \quad \frac{k_{d}}{4 b d_{M}}>\varepsilon_{2}^{2}
$$

which hold in view of (12a), since $\varepsilon_{1}, \varepsilon_{2}<1$. It is also clear from Assumption 1.2, that $V$ is proper since $D$ is bounded. Using (21) and (26) we see that $v(t):=V(t, x(t))$ satisfies

$$
\dot{v}(t) \leq-\varepsilon_{1} k_{p}|\tilde{q}(t)|^{2}+c_{1}^{\prime}|\tilde{q}(t)|+c_{2}^{\prime}
$$

for all $x(t) \in \Omega, t \in\left[\tau, t^{\max }\right)$ and appropriate (innocuous) values of $c_{1}^{\prime}$ and $c_{2}^{\prime}$. If $|\tilde{q}(t)|$ grows unboundedly, there exists $t \in\left[\tau, t^{\max }\right.$ ) (if necessary, replace $t^{\max }$ with $t_{\text {new }}^{\max }>t^{\max }$ ) such that $\dot{v}(t) \leq 0$. By continuity we may extend $\left[\tau, t^{\max }\right)$ to $[\tau, \infty)$ and conclude that $v(t)$ is uniformly bounded. Since $x \mapsto V$ is proper $|x(t)|$ is also uniformly bounded on $[\tau, \infty)$. Using forward completeness again, we obtain uniform global boundedness on $\left[t_{0}, \infty\right)$.

This completes the proof of the proposition.

The standing assumption in the following proposition is that the solutions are uniformly globally bounded, which has been established above.

Proposition 2 Consider the system (1) under the conditions of Theorem 3. Assume that for each $r>0$ there exists $R(r)$ such that if $x\left(t_{\circ}\right) \in B_{r}$ then $x(t) \in B_{R}$ for all $t \geq t_{\circ}$. Under these conditions, the origin is uniformly globally attractive.

Proof. Let the control gains be fixed according to (12). Consider a function $V: \mathbb{R}_{>0} \times B_{R} \rightarrow \mathbb{R}$ defined as in (27). Under Assumption 1 its total time-derivative along the trajectories of (14), (15) satisfies, for all $(t, x) \in \mathbb{R}_{\geq 0} \times B_{R}$,

$$
\begin{aligned}
& \dot{V} \leq-\frac{\varepsilon_{1} k_{p}|\tilde{q}|^{2}}{4}-\frac{\varepsilon_{2} b d_{m}|\dot{\tilde{q}}|^{2}}{8}-\left[\frac{\varepsilon_{2} b d_{m}}{8}-\varepsilon_{1} d_{M}\right]|\dot{\tilde{q}}|^{2} \\
& -\frac{1}{2}\left[\begin{array}{c}
|\tilde{q}| \\
|\dot{\tilde{q}}|
\end{array}\right]^{\top}\left[\begin{array}{cc}
\varepsilon_{1} k_{p} / 2 & -\varepsilon_{1} k_{c}\left(R+k_{\delta}\right) \\
* & \varepsilon_{2} b d_{m} / 2
\end{array}\right]\left[\begin{array}{c}
|\tilde{q}| \\
|\dot{\tilde{q}}|
\end{array}\right] \\
& -\frac{1}{2}\left[\begin{array}{c}
|\tilde{q}| \\
|\vartheta|
\end{array}\right]^{\top}\left[\begin{array}{cc}
\varepsilon_{1} k_{p} / 2 & -\left(\varepsilon_{1} k_{d}+\varepsilon_{2} k_{p}\right) \\
* & k_{d} a / 2 b
\end{array}\right]\left[\begin{array}{c}
|\tilde{q}| \\
|\vartheta|
\end{array}\right] \\
& -\frac{1}{2}\left[\begin{array}{c}
|\dot{\tilde{q}}| \\
|\vartheta|
\end{array}\right]^{\top}\left[\begin{array}{cc}
\frac{\varepsilon_{2} b d_{m}}{2} & -\varepsilon_{2}\left(k_{c}\left(R+k_{c} k_{\delta}\right)+a d_{M}\right) \\
* & k_{d} a / b
\end{array}\right]\left[\begin{array}{c}
|\dot{\tilde{q}}| \\
|\vartheta|
\end{array}\right] \\
& -\left(\frac{k_{d} a}{4 b}-\varepsilon_{2} k_{d}\right)|\vartheta|^{2}+k_{c} k_{\delta}|\dot{\tilde{q}}|^{2}
\end{aligned}
$$

where "*" stands for the opposite element in the matrix with respect to the main diagonal. The factor of $-|\dot{\tilde{q}}|^{2}$ in the third term above is positive, as well as the first matrix above is positive definite, if

$$
\frac{\varepsilon_{2}}{4 \varepsilon_{1}} b d_{m} \geq \frac{k_{c}^{2}\left(R+k_{\delta}\right)^{2}}{k_{p}}+2 d_{M}
$$

which holds for control gains independent of the initial conditions and of $R$, if

$$
\frac{\varepsilon_{2}}{\varepsilon_{1}}=\mathcal{O}\left(R^{2}\right)
$$

The second matrix is positive if

$$
\frac{\varepsilon_{1} k_{p} k_{d} a}{4 b} \geq\left(\varepsilon_{1} k_{d}+\varepsilon_{2} k_{p}\right)^{2}
$$

which holds for sufficiently small values of $\varepsilon_{1}$ and $\varepsilon_{2}$. Finally, the third matrix is positive definite if

$$
\frac{k_{d} a d_{m}}{4} \geq \varepsilon_{2}\left[\left(R+k_{c} k_{\delta}\right) k_{c}+k_{\delta} k_{c}+a d_{M}\right]^{2}
$$

which holds for sufficiently small values of

$$
\varepsilon_{2}=\mathcal{O}\left(\frac{1}{R^{2}}\right)
$$

which in turn, in view of (29), imposes that

$$
\varepsilon_{1}=\mathcal{O}\left(\frac{1}{R^{4}}\right) \text {. }
$$

Hence, there exists $c>0$ such that

$$
\dot{V}(t, x) \leq-c|x|^{2}+k_{c} k_{\delta}|\dot{\tilde{q}}|^{2} \quad \forall(t, x) \in \mathbb{R}_{\geq 0} \times B_{R} .
$$

Now let the property of uniform global boundedness generate a number $r>0$ such that $x\left(t_{\circ}\right) \in B_{r}$ implies that $x(t) \in B_{R}$ for all $t \geq t_{\circ}$ and for any $t_{\circ} \geq 0$. From (32) we have

$$
\dot{v}(t) \leq-\left[c|x(t)|^{2}-\nu\right] \quad \forall\left(t, x_{\circ}\right) \in \mathbb{R}_{\geq 0} \times B_{r} .
$$

where $\nu=k_{c} k_{\delta} R^{2}$. The claim follows observing that the previous development holds for arbitrary $r>0, \nu>0$, integrating on both sides of (33) and invoking Lemma 1 with $\gamma(s)=c|s|^{2}$.

To the best of our knowledge, constructing a strict (control) Lyapunov function is an open problem which is illustrated by but not limited to the case of the controller (13). In a general nonlinear context, the state of the art in constructing Lyapunov functions for nonlinear time-varying systems relies on Lyapunov functions that have negative semi-definite derivatives — see [14]. That is, in the present context, the methods in the latter reference require that $\dot{V}_{1} \leq 0$ as opposed to (17).

\section{CONCLUSIONS}

The problem solved in this paper may not be overestimated; Euler-Lagrange systems are of a special kind in the sense that they belong to the class of systems studied in the seminal paper [17] for which it is proved that global output feedback stabilization is impossible, if not for the structural property that the Coriolis forces (the highly nonlinear terms) produce no work. We believe that the method of proof used here may unlock the path to solutions 
to other problems such as global proportional-integralderivative control ${ }^{3}$. Further research is also undergoing to construct a control Lyapunov function with aim at realizing an to as adaptive version of the controller presented here.

\section{Acknowledgements}

The author is indebted to R. Ortega and E. Panteley for the arbitrarily large (but bounded) number of discussions on this problem during the last 20 and 15 years respectively, not withstanding this note.

\section{REFERENCES}

[1] G. Besançon. Contributions à l'étude et à l'observation des systèmes non linéaires avec recours au calcul formel. PhD thesis, INPG, November 1996.

[2] G. Besançon. Global output feedback tracking control for a class of lagrangian systems. Automatica, 36(12):1915-1921, 2000.

[3] E. Børhaug and K. Y. Pettersen. Global output feedback pid control for n-dof euler-lagrange systems. In Proc. IEEE American Control Conference, pages 4993-4999, Minneapolis, Minnesota, USA, 2006.

[4] I. V. Burkov. Mechanical system stabilization via differential observer. In IFAC Conference on System Structure and Control, pages 532-535, Nantes, France, 1995.

[5] T. I. Fossen. Guidance and control of ocean vehicles. John Wiley \& Sons Ltd., 1994.

[6] W. Hahn. Stability of motion. Springer-Verlag, New York, 1967.

[7] K. Kalsi, J. Lian, S. Hui, and H. Zak. Sliding-mode observers for systems with unknown inputs: A high-gain approach. Automatica, 46:347-353, 2010.

[8] R. Kelly. A simple set-point robot controller by using only position measurements. In Proc. 12th. IFAC World Congress, volume 6, pages 173-176, Sydney, Australia, 1993.

[9] D. E. Koditschek. Application of a new Lyapunov function to global adaptive attitude tracking. In Proc. 27th. IEEE Conf. Decision Contr., Austin, TX, 1988.

[10] A. Loría. Global tracking control of one degree of freedom EulerLagrange systems without velocity measurements. European J. of Contr., 2(2), 1996.

[11] A. Loría and K. Melhem. Position feedback global tracking control of EL systems : a remodelling approach. IEEE Trans. on Automat. Contr., 47(5):841-848, 2002.

[12] A. Loría and R. Ortega. On tracking control of rigid and flexible joints robots. Appl. Math. and Comp. Sci., special issue on Mathematical Methods in Robotics, K. Tchon and A. Gosiewsky, eds, 5(2):101-113, 1995.

[13] A. Loría and E. Panteley. A separation principle for Euler-Lagrange systems, volume 244 of Lecture Notes in Control and Information Sciences, chapter in New directions in nonlinear observer design. Springer Verlag, H. Nijemeijer, T. I. Fossen, eds., London, 1999.

[14] M. Malisoff and F. Mazenc. Constructions of Strict Lyapunov functions. Springer Verlag, London, 2009.

[15] R. Marino and P. Tomei. Global adaptive output feedback control of nonlinear systems. Part I : Linear parameterization. IEEE Trans. on Automat. Contr., 38:17-32, 1993.

[16] Riccardo Marino and Patrizio Tomei. Dynamic output feedback linearization and global stabilization. Syst. \& Contr. Letters, 17(2):115 $-121,1991$.

[17] F. Mazenc, L. Praly, and W. P. Dayawansa. Global stabilization of output feedback: examples and counterexamples. Syst. \& Contr. Letters, 22:119-125, 1994.

[18] E. V. L. Nunes and L. Hsu. Global tracking for robot manipulators using a simple causal pd controller plus feedforward. Robotica, 2009.

[19] R. Ortega, A. Loría P. J. Nicklasson, and H. Sira-Ramírez. Passivitybased Control of Euler-Lagrange Systems: Mechanical, Electrical and Electromechanical Applications. Series Comunications and Control Engineering. Springer Verlag, London, 1998. ISBN 1-85233-016-3.

[20] B. Paden and R. Panja. Globally asymptotically stable PD+ controller for robot manipulators. Int. J. of Contr., 47:1697-1712, 1988.

${ }^{3}$ We refer to the classical PID control and not to available nonlinear variants of the latter.
[21] E. Panteley, A. Loría and A. Teel. Relaxed persistency of excitation for uniform asymptotic stability. IEEE Trans. on Automat. Contr., 46(12):1874-1886, 2001.

[22] K. P. Persidskiĭ. Ob ustoichivosti dvizhenya po pervomu priblizheniyu. Mat. Sbornik, 40(3):284-293, 1933. English title: On the stability of motion in the first aproximation.

[23] J. G. Romero. Robust Energy Shaping Control of Nonlinear Systems. $\mathrm{PhD}$ thesis, Univ Paris Sud, Feb. 2013.

[24] R. Sepulchre, M. Janković, and P. Kokotović. Constructive nonlinear control. Springer Verlag, 1997.

[25] M. W. Spong. Remarks on robot dynamics:canonical transformations and riemannian geometry. In Proc. IEEE Conf. Robotics Automat., pages 554-559, Nice, France, 1992.

[26] M. Vidyasagar. Nonlinear systems analysis. Prentice Hall, New Jersey, 1993.

[27] E. Zergeroglu, D. M. Dawson, M. S. de Queiroz, and M. Krstić. On global output feedback tracking control of robot manipulators. In Proc. 39th. IEEE Conf. Decision Contr., pages 5073-5078, Sydney, Australia, 2000. 